\title{
Validation of MR-Based Attenuation Correction of a Newly Released Whole-Body Simultaneous PET/MR System
}

\author{
Guobing Liu $\mathbb{D},{ }^{1,2,3}$ Tuoyu Cao, ${ }^{4}$ Lingzhi Hu, ${ }^{4}$ Jiaxu Zheng, ${ }^{4}$ Lifang Pang, ${ }^{1,2,3}$ \\ Pengcheng $\mathrm{Hu}\left(\mathbb{0},{ }^{1,2,3}\right.$ Yushen $\mathrm{Gu}^{1,2,3}$ and Hongcheng Shi $\mathbb{\circledR}^{1,2,3}$ \\ ${ }^{1}$ Department of Nuclear Medicine, Zhongshan Hospital, Fudan University, Shanghai 200032, China \\ ${ }^{2}$ Shanghai Institute of Medical Imaging, Shanghai 200032, China \\ ${ }^{3}$ Institute of Nuclear Medicine, Fudan University, Shanghai 200032, China \\ ${ }^{4}$ Shanghai United Imaging Healthcare Co., Ltd., Shanghai 201807, China
}

Correspondence should be addressed to Hongcheng Shi; shihongcheng163@163.com

Received 1 May 2019; Accepted 22 October 2019; Published 28 November 2019

Academic Editor: Jiun-Jie Wang

Copyright (C) 2019 Guobing Liu et al. This is an open access article distributed under the Creative Commons Attribution License, which permits unrestricted use, distribution, and reproduction in any medium, provided the original work is properly cited.

The aim of this study was to validate quantitative performance of a newly released simultaneous positron emission tomography (PET)/magnetic resonance imaging (MRI) scanner, by using MR-based attenuation correction (MRAC), both in phantom study and in patient study. PET/MRI image uniformities of a phantom under different hardware configurations were tested and compared. Thirty patients were examined with 2-deoxy-2- $\left[{ }^{18} \mathrm{~F}\right]$ fluoro-D-glucose $\left({ }^{18} \mathrm{~F}\right.$-FDG) PET/computed tomography (CT) and

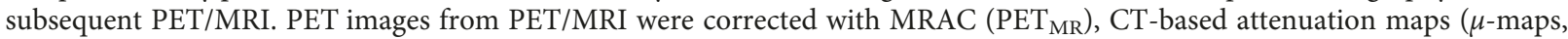

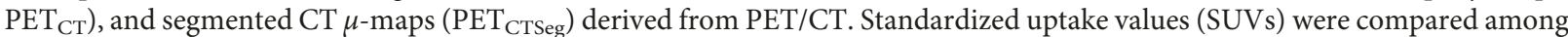
the 3 sets of PET in main organs (bone, liver and lung) and in 52 FDG-avid lesions, including soft-tissue lesions and bone lesions. The result showed that PET imaging uniformities of PET/MRI under different configurations were good $(<8.8 \%)$. The SUV differences among the 3 sets of PET varied with organs and lesion types. In detail, the mean relative differences of SUV between $\mathrm{PET}_{\mathrm{MR}}$ and $\mathrm{PET}_{\mathrm{CT}}$ were as follows: $-18.8 \%$, bone $\left(\mathrm{SUV}_{\text {mean }}\right) ;-8.0 \%$, liver $\left(\mathrm{SUV}_{\text {mean }}\right) ;-12.2 \%$, lung $\left(\mathrm{SUV}_{\text {mean }}\right) ;-18.1 \%$, bone lesions $\left(\mathrm{SUV}_{\text {mean }}\right) ;-13.3 \%$, bone lesions $\left(\mathrm{SUV}_{\max }\right) ;-8.2 \%$, soft-tissue lesions $\left(\mathrm{SUV}_{\text {mean }}\right)$; and $-7.3 \%$, soft-tissue lesions $\left(\mathrm{SUV}_{\text {max }}\right)$. The mean relative differences between $\mathrm{PET}_{\mathrm{MR}}$ and $\mathrm{PET}_{\mathrm{CTSeg}}$ were as follows: $-19.0 \%$, bone $\left(\mathrm{SUV}_{\text {mean }}\right) ;-3.5 \%$, liver $\left(\mathrm{SUV}_{\text {mean }}\right)$; $-3.3 \%$, lung $\left(\mathrm{SUV}_{\text {mean }}\right) ;-19.3 \%$, bone lesions $\left(\mathrm{SUV}_{\text {mean }}\right) ;-17.5 \%$, bone lesions $\left(\mathrm{SUV}_{\max }\right) ;-5.5 \%$, soft-tissue lesions $\left(\mathrm{SUV}_{\text {mean }}\right)$; and $-4.4 \%$, soft-tissue lesions $\left(\mathrm{SUV}_{\max }\right)$. The differences of SUV between $\mathrm{PET}_{\mathrm{MR}}$ and $\mathrm{PET}_{\mathrm{CT}}$ were larger than those between $\mathrm{PET}_{\mathrm{MR}}$ and $\mathrm{PET}_{\mathrm{CTSeg}}$, in both soft tissue and soft-tissue lesions $(P<0.001)$, but not in bone or bone lesions. In conclusion, MRAC in the newly released PET/MR system is accurate in most tissues, with SUV deviations being generally less than $10 \%$, compared to $\mathrm{PET} / \mathrm{CT}$. In bone, however, underestimations can be substantial, which may be partially attributed to segmentation of the MRbased $\mu$-maps.

\section{Introduction}

The integrated whole-body positron emission tomography $(\mathrm{PET}) /$ magnetic resonance imaging (MRI) is emerging as a potential tool in clinical practice and in medical research. To date, there have been three commercial PET/MRI systems introduced; the first system was launched by Siemens (mMR PET/MR) in 2010 featuring an integrated design for simultaneous acquisition, the second system was released by Philips (Ingenuity TF PET/MR) in 2011 using a rotating table for sequential acquisitions, and most recently, a second simultaneous PET/MR system was introduced by GE (SIGNA PET/MR) in 2016 [1-3].

In 2018, the United Imaging Healthcare Corporation (Shanghai, China) released a fourth system (uPMR790) to be sold commercially. This system comprises a $3 \mathrm{~T}$ superconducting magnet, a gradient system with a set of secondorder active shimming coil $(50 \mathrm{mT} / \mathrm{s}, 200 \mathrm{~T} / \mathrm{m} / \mathrm{s})$, and a 48 channel radio frequency (RF) receiving system. The PET detector is installed between the gradient coil and body coil, 
which comprises 20 modules with a transverse field of view (FOV) of $60 \mathrm{~cm}$ and an axial FOV of $32 \mathrm{~cm}$. Each module contains $5 \times 14$ blocks, and each block has 4 SiPM detector channels coupled with a $7 \times 8$ array of $15.5 \times 2.76 \times 2.76 \mathrm{~mm}^{3}$ LYSO crystals through the proprietary design of internal light guide. The entire system comprises 112 rings, and each ring contains 700 crystal channels, making 78400 crystal channels in total. In short, the uPMR790 features $2.8 \mathrm{~mm}$ PET spatial resolution, the highest resolution to date, and the longest axial FOV $(32 \mathrm{~cm})$ of all PET/MR systems. The PET component of PET/MR systems remains challenging due to hardware integrations, and aspects of PET image reconstruction, especially attenuation correction, still remain as an issue. Therefore, performance validation of this system is needed before putting it into clinical use.

Interpretation of the PET images always requires quantification of the tracer distribution, which heavily relies on attenuation correction (AC) during image reconstruction. However, the MR-based AC (MRAC) is not straightforward and quite different from computed tomography- (CT-) based AC (CTAC), as signal intensity of MRI does not reflect the electron density of the tissue, rendering a direct transformation of signal intensity to linear ACs (LACs) impossible [4-6]. To solve this problem, several approaches have been developed for MRAC, of which the segmentation-based methods have been commonly used and implemented into vendor-provided software [5, 7]. These methods separate the human body into several different tissue types usually using a T1-weighted 3-dimensional gradient-echo sequence with 2 echoes for fat and water separation. Afterward, predefined LACs are used for the different tissue types, allowing for computation of an attenuation map ( $\mu$-map) $[5,6,8]$.

Several studies compared the segmentation-based MRAC against the well-established CTAC [7, 9-16]. However, conflicting results regarding underestimation or overestimation in SUVs of certain tissues remain. Furthermore, all of these comparisons were performed directly between the segmented MRAC and the continuous CTAC. Therefore, the results and comparisons inevitably suffered from the impact of segmentation to the MRAC data. In addition, nonpatient objects such as MR coils within the PET FOV could also affect the accuracy of attenuation correction $[9,15,17]$. Therefore, it is necessary to exclude the impact from these factors before validating the accuracy of the MRAC method.

This study aims at validating the performance of a simultaneous whole-body PET/MR system through two steps. In the first step, we investigated the impact of the hardware components of PET/MRI scanner (i.e., the track, patient bed, and MR coils) on the MRAC method based on the phantom study. In the second step, a volume-of-interest (VOI) based approach was conducted to validate the quantification accuracy of the MRAC-based PET in different tissues and lesions through patient-based study.

\section{Materials and Methods}

2.1. Phantom Study. A uniform cylinder phantom $(20 \mathrm{~cm}$ in diameter) filled with $1 \mathrm{mCi}{ }^{68} \mathrm{Ge}$ was imaged at the center of a whole-body simultaneous PET/MR system (uPMR 790, United Imaging Healthcare, Shanghai, China). Data were acquired under four different configurations (Figure 1): (1) with the track of patient bed; (2) with the track, patient bed, and the spine coil; (3) with the track, patient bed, spine coil, and the base of the head coil; (4) with the track, patient bed, spine coil, and the whole head coil. PET data were acquired for 6 minutes under each configuration and were reconstructed by using the algorithm of time of flight (TOF) ordered subset expectation maximization (OSEM), with 20 subsets, 3 iterations, image matrix of $256 \times 256$, voxel size of $2.4 \times 2.4 \times 2.85 \mathrm{~mm}^{3}$, and a $3 \mathrm{~mm}$ Gaussian filter.

The attenuation maps for rigid objects were obtained from CT scans. It is also crucial for determining the relative position between these objects and the imaging FOV. The position of the track was fixed, so its attenuation map was hardcoded into reconstruction. The positions of patient bed and coils were determined by the axial position of patient bed since coils had a fixed position on patient bed. The position of the phantom was determined with an automatic coregistration process between the build-in model and the non-attenuation-corrected PET image.

The uniformity of the image was calculated based on the following approach. Five slices located at the center, $\pm 3 \mathrm{~cm}$, and $\pm 6 \mathrm{~cm}$ of the image were selected from the image volume. For each slice, mean SUVs from four circles with diameters of $60 \mathrm{~mm}$ and a circle with a diameter of $120 \mathrm{~mm}$ were measured, denoted as $B 1, B 2, B 3, B 4$, and $A 1$, respectively (Figure 2). The slice uniformity is defined as

$$
\text { slice_uniformity }=\max \left(\left|\frac{\max (B 1, B 2, B 3, B 4)-A 1}{A 1}\right|,\left|\frac{\min (B 1, B 2, B 3, B 4)-A 1}{A 1}\right|\right) \text {. }
$$

The whole image uniformity is defined as the maximum of the slice uniformities from the five slices.

\section{Patient Study}

3.1. Ethical Statement. The patient study was retrospective and was approved by the Institutional Review Board (IRB)/
Ethics Committee (IRB 88000039-QCN-CT5-01) in accordance with the ethical standards as laid down in the 1964 Declaration of Helsinki and its later amendments. Informed consent was obtained from all patients.

3.2. Patient Cohort. Thirty patients referred for 2-deoxy-2-

$\left[{ }^{18} \mathrm{~F}\right]$ fluoro-D-glucose (FDG) PET/CT scans were included. 


\begin{tabular}{|c|c|c|c|c|}
\hline & Case 1 & Case 2 & Case 3 & Case 4 \\
\hline Scan setup & $\begin{array}{c}\text { Cylinder phantom + } \\
\text { Track }\end{array}$ & $\begin{array}{c}\text { Cylinder phantom }+ \\
\text { Bed }+ \text { Spine coil }+ \\
\text { Track }\end{array}$ & $\begin{array}{c}\text { Cylinder phantom }+ \\
\text { Bed }+ \text { Spine coil }+ \\
\text { Head coil base }+ \\
\text { Track }\end{array}$ & $\begin{array}{c}\text { Cylinder phantom }+ \\
\text { Bed }+ \text { Spine coil }+ \text { Head } \\
\text { coil base }+ \text { Head coil top } \\
+ \text { Track }\end{array}$ \\
\hline $\begin{array}{l}\text { Hardware } \\
\mu \text {-map } \\
\text { template }\end{array}$ & Track & $\begin{array}{c}\text { Bed }+ \text { Spine coil }+ \\
\text { Track }\end{array}$ & $\begin{array}{c}\text { Bed }+ \text { Spine coil }+ \\
\text { Head coil base }+ \\
\text { Track }\end{array}$ & $\begin{array}{l}\text { Bed }+ \text { Spine coil }+ \\
\text { Head coil base }+ \text { Head } \\
\text { coil top }+ \text { Track }\end{array}$ \\
\hline \multicolumn{5}{|l|}{$\begin{array}{l}\text { Combined } \\
\mu \text {-map }\end{array}$} \\
\hline Uniformity & $8.24 \%$ & $8.78 \%$ & $5.02 \%$ & $7.60 \%$ \\
\hline PET image & & & & \\
\hline
\end{tabular}

FIgURE 1: Image uniformity under different MR hardware configurations.

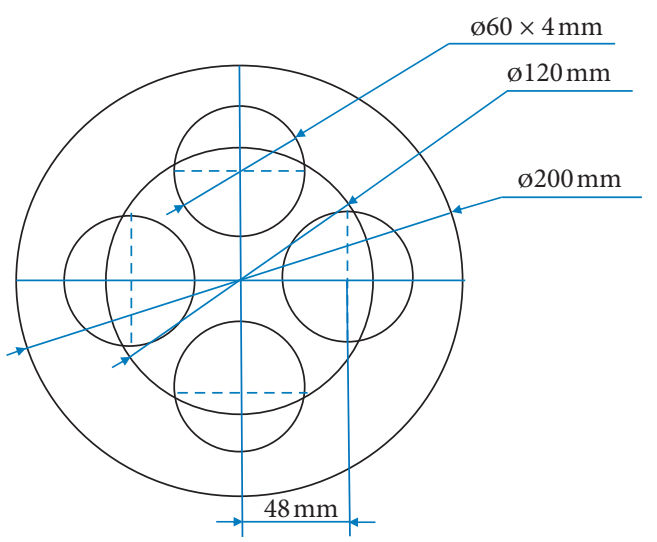

(a)

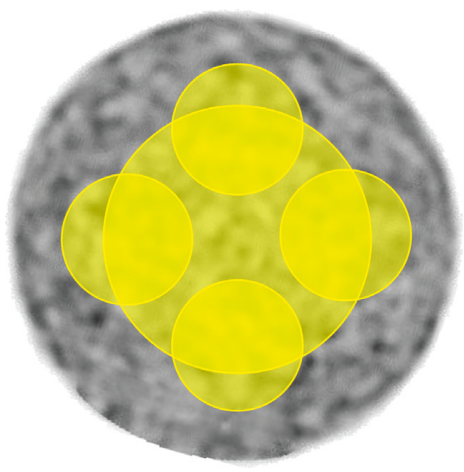

(b)

FIGURE 2: Schematic diagram showing region of interest drawing in cylinder phantom for uniformity calculation (a). Uniformity calculation on a PET image (b).

Inclusion criteria were as follows: (1) patients proven to have or suspected of having malignancy, with an indication for PET/CT for diagnosis, staging or restaging, follow-up, and therapy-response evaluation; (2) medical conditions of patients were stable. Exclusion criteria were as follows: (1) examinations were only on one bed position (e.g., head or abdomen); (2) obvious metal artifacts; (3) technical problems (e.g., patient movement); and (4) MR-CT registration failure. The basic information of patients is summarized in Table 1.

3.3. PET/CT Imaging. All patients fasted for at least 6 hours, and serum glucose was checked before the injection of FDG. The amount of injected radioactivity was calculated by measuring the radioactivity of the syringe before and after injection. The mean injected dose was $310.8 \mathrm{MBq}$ (standard deviation (SD), 63.6). One hour after injection, $\mathrm{PET} / \mathrm{CT}$ scanning was performed from the skull base to the proximal thigh in a supine position with arms over head, on the uMI 780 PET/CT scanner (United Imaging Healthcare, Shanghai, China) which had a similar PET configuration with the uPMR 790 PET/MRI scanner. Helical CT acquisition was performed without contrast enhancement using the following parameters: tube current, $274 \mathrm{mAs}$; tube voltage, $120 \mathrm{kV}$; collimation configuration, $80 \times 0.5 \mathrm{~mm}$; pitch, 0.516; matrix size, $512 \times 512$; and scanning time, 0.8 seconds per rotation. PET was acquired for 2 minutes per bed position in a three-dimensional mode, and images were reconstructed by using the OSEM algorithm. The image matrix was $256 \times 256$, corresponding to a $3 \mathrm{~mm}$ inplane pixel size with a plane thickness of $3 \mathrm{~mm}$. 
TABLE 1: Basic information of patients.

\begin{tabular}{|c|c|c|c|c|c|c|c|c|}
\hline Patient & Sex & $\begin{array}{c}\text { Age } \\
\text { (years) }\end{array}$ & $\begin{array}{l}\text { Height } \\
(\mathrm{cm})\end{array}$ & $\begin{array}{l}\text { Weight } \\
(\mathrm{kg})\end{array}$ & Diagnosis & $\begin{array}{l}\text { Dose } \\
(\mathrm{mCi})\end{array}$ & $\begin{array}{c}\text { Duration of PET/MRI after } \\
\text { injection (min) }\end{array}$ & $\begin{array}{c}\text { Clinical } \\
\text { indication }\end{array}$ \\
\hline 1 & $\mathrm{~F}$ & 55 & 162.0 & 62.0 & Lung cancer & 7.39 & 61 & $\begin{array}{l}\text { Follow- } \\
\text { up }\end{array}$ \\
\hline 2 & $\mathrm{~F}$ & 71 & 152.0 & 70.0 & Cerebral metastasis & 8.33 & 98 & Diagnosis \\
\hline 3 & M & 57 & 172.0 & 68.0 & Rectal cancer & 8.65 & 114 & Restaging \\
\hline 4 & M & 65 & 180.0 & 87.5 & Lymphoma & 10.49 & 99 & Staging \\
\hline 5 & M & 64 & 170.0 & 64.0 & Esophageal cancer, lung cancer & 8.22 & 104 & Staging \\
\hline 6 & M & 66 & 167.0 & 76.0 & Pancreatic cancer & 9.52 & 146 & Staging \\
\hline 7 & M & 17 & 174.0 & 72.0 & Thyroid cancer & 8.60 & 94 & Diagnosis \\
\hline 8 & M & 69 & 162.0 & 67.0 & Enterocoelic sarcoma & 8.65 & 120 & Staging \\
\hline 9 & $\mathrm{~F}$ & 73 & 157.0 & 63.0 & Ovarian cancer & 7.05 & 96 & Staging \\
\hline 10 & $\mathrm{~F}$ & 50 & 165.0 & 54.0 & Pancreatic cancer & 6.99 & 87 & Staging \\
\hline 11 & $\mathrm{~F}$ & 65 & 163.0 & 58.0 & Cholangiocarcinoma & 6.87 & 101 & Staging \\
\hline 12 & $\mathrm{M}$ & 66 & 164.0 & 51.0 & Esophageal cancer & 6.13 & 102 & Staging \\
\hline 13 & $\mathrm{~F}$ & 51 & 161.0 & 67.0 & Rectal cancer & 8.01 & 90 & TRE \\
\hline 14 & $\mathrm{~F}$ & 68 & 161.0 & 63.0 & Lung cancer & 7.33 & 87 & Staging \\
\hline 15 & $\mathrm{M}$ & 46 & 170.0 & 105.0 & Liver cancer & 13.61 & 162 & Diagnosis \\
\hline 16 & M & 62 & 175.0 & 75.0 & Lung cancer & 9.33 & 88 & Staging \\
\hline 17 & $\mathrm{M}$ & 70 & 170.0 & 70.0 & Duodenal cancer & 8.82 & 96 & Staging \\
\hline 18 & M & 70 & 162.0 & 69.0 & Lung cancer & 8.20 & 119 & Staging \\
\hline 19 & M & 64 & 164.0 & 61.0 & Colon cancer & 8.50 & 117 & Staging \\
\hline 20 & M & 63 & 168.0 & 68.0 & Gastric cancer & 8.57 & 94 & TRE \\
\hline 21 & M & 65 & 172.0 & 71.0 & Colon cancer & 8.85 & 107 & Staging \\
\hline 22 & M & 69 & 170.0 & 72.0 & Pancreatic cancer & 9.99 & 115 & Diagnosis \\
\hline 23 & M & 54 & 151.0 & 52.0 & Liver cancer & 6.40 & 66 & Staging \\
\hline 24 & M & 46 & 173.0 & 84.0 & Liposarcoma in teres major & 10.71 & 103 & Staging \\
\hline 25 & M & 60 & 171.0 & 73.8 & Liver cancer & 3.64 & 94 & TRE \\
\hline 26 & M & 75 & 173.0 & 74.0 & Liver cancer & 9.38 & 107 & Restaging \\
\hline 27 & $\mathrm{~F}$ & 60 & 156.0 & 56.0 & Duodenal cancer & 6.69 & 114 & Staging \\
\hline 28 & M & 51 & 174.0 & 85.0 & Liver cancer & 10.08 & 93 & Diagnosis \\
\hline 29 & M & 61 & 172.0 & 70.5 & Lymphoma & 8.45 & 82 & Diagnosis \\
\hline 30 & $\mathrm{~F}$ & 69 & 156.0 & 60.0 & Liver cancer & 8.63 & 95 & Diagnosis \\
\hline Mean & & 60.7 & 166.2 & 69.0 & & 8.40 & 101.7 & \\
\hline $\mathrm{SD}$ & & 11.2 & 7.1 & 11.1 & & 1.72 & 19.6 & \\
\hline
\end{tabular}

Note: M, male; F, female; SD, standard deviation; TRE, therapy-response evaluation.

3.4. PET/MR Imaging. After PET/CT imaging, all patients experienced simultaneous PET/MR imaging on the uPMR 790 PET/MRI system (United Imaging Healthcare, Shanghai, China), composed of a 3.0-Tesla MR imager and a fully integrated PET detector. Mean time intervals between PET/CT and PET/MR imaging was 45.4 (SD 16.2) minutes, while mean time intervals between FDG injection and PET/MR imaging was 101.7 (SD, 19.6) minutes (Table 1). For MRAC, a 3D T1-weighted spoiled gradient-echo sequence with Dixonbased water-fat separation imaging (WFI) was acquired in coronal plane with a repetition time of $4.6 \mathrm{~ms}$, an echo time of $3.2 \mathrm{~ms}$, a slice thickness of $2.4 \mathrm{~mm}$, an FOV of $500 \times 350 \mathrm{~mm}$, a matrix of $206 \times 144$, and an acquisition time of 31 seconds. Compressed sensing-based technology was used to speed up the acquisition. Tissue segmentation and $\mu$-map calculation were carried out automatically by the vendor-provided algorithm. Whole-body PET data were acquired in $3 \mathrm{D}$ list mode. Four bed positions with an average time of 6 minutes per bed were set to cover the area from skull base to proximal thigh of patients with an overlap of $30 \%$.

\section{PET Attenuation Correction}

4.1. MR-Based Attenuation Correction. The WFI images were preprocessed to correct for the bias field signal before submitting to segmentation. Then, the corrected images were segmented into four classes: soft tissue, fat, lung, and air according to predetermined attenuation coefficients, namely, $0.096 \mathrm{~cm}^{-1}, 0.080 \mathrm{~cm}^{-1}, 0.032 \mathrm{~cm}^{-1}$, and $0 \mathrm{~cm}^{-1}$, in order. A deep learning-based technique was used for lung segmentation so that the segmentation could be done with arbitrary bed positions without users' input [18]. A U-Net model was used, and training data were made by arbitrarily cropping whole-body DIXON in-phase images with lung region labeled [19]. Furthermore, truncation completion was achieved with the contour of arms from PET images by segmenting non-attenuation-corrected PET images. PET images were reconstructed with a standard process provided by the vendor as follows: TOF-OSEM with 20 subsets, 3 iterations, image matrix of $256 \times 256$, voxel size of $2.4 \times 2.4 \times 2.85 \mathrm{~mm}^{3}$, and $3 \mathrm{~mm}$ post-Gaussian filter. 
4.2. CT-Based Attenuation Correction. In order to create CT $\mu$-maps for PET reconstruction, the CT images were coregistered to the PET/MR space with a nonrigid registration algorithm using the publicly available software-Elastix [20]. The set of parameters were carefully adjusted so that the coregistration performance was optimized. We would like to point out that since nonrigid registration is underdetermined by nature, the algorithm primarily focused on good alignment in tissue boundaries between two modalities. Since CT images were acquired with arms up while MR images were acquired with arms down, the arms were excluded in the coregistration process with body-arm boundaries manually drawn for each case (Figure 3). After the coregistration process, the arms from MRAC were stitched back to CTAC to form a complete attenuation map. For each patient, two sets of CT $\mu$-maps were obtained. One was derived directly from CT images with linear transformation. The other was segmented CT $\mu$-maps, which was calculated as follows: for voxels of LAC $<0.003 \mathrm{~mm}^{-1}$, they were assigned $\mathrm{LAC}=0 \mathrm{~mm}^{-1}$ (air); for voxels of LAC $0.003-0.009 \mathrm{~mm}^{-1}$, they were assigned $\mathrm{LAC}=0.008 \mathrm{~mm}^{-1}$ (fat); for voxels of LAC $0.009-0.011 \mathrm{~mm}^{-1}$, they were assigned $\mathrm{LAC}=0.0096 \mathrm{~mm}^{-1}$ (water); for voxels of LAC $>0.011 \mathrm{~mm}^{-1}$, they were assigned LAC $=0.0161 \mathrm{~mm}^{-1}$ (bone); and for lung regions, they were assigned $\mathrm{LAC}=0.0032 \mathrm{~mm}^{-1}$. The CTAC maps were used in the same manner as MRAC maps in the PET reconstruction process.

In summary, the PET dataset from PET/MRI was reconstructed using the same parameters with the $M R$ $\mu$-map $\left(\mathrm{PET}_{\mathrm{MR}}\right)$, the CT $\mu$-map ( $\left.\mathrm{PET}_{\mathrm{CT}}\right)$, and the segmented CT $\mu$-map $\left(\mathrm{PET}_{\mathrm{CTSeg}}\right)$, as shown in Figure 4.

4.3. PET Quantification Analysis. Quantification analyses were conducted on the vendor-provided image viewer. For measuring SUVs on the 3 sets of PET data with the same regions of interest (ROIs), the Dixon T1 images were selected and registered with all of the PET data, respectively, for providing anatomic information. ROIs in diameters of $2 \mathrm{~cm}$ were drawn in homogeneous area of main organs-the right lobe of the liver, the right lower lobe of the lung, and the fourth lumbar vertebrae, and SUVs were measured. Care was taken to avoid placing ROIs on large vessels, organ borders, lesions, and close to the border of the PET FOV. In addition, for analyzing the accuracy of MRAC on lesion basis, 52 FDGavid lesions from 30 patients were selected and grouped into two parts: (1) lesions located within or around bone $(n=28)$ and (2) lesions located in soft tissue $(n=24)$. VOIs with a $50 \%$ isocontour of $\mathrm{SUV}_{\max }$ were drawn around lesions. Mean SUVs $\left(S_{\text {mean }}\right)$ were measured for major organs; while both $\mathrm{SUV}_{\text {mean }}$ and maximum SUV (SUV $\mathrm{Sax}_{\text {max }}$ ) were measured for lesions. This work was performed by a nuclear medicine physician with experience over 15 years.

4.4. Statistical Analysis. Statistical analyses were performed using SPSS 20.0 (SPSS Statistics; IBM, Armonk, NY). Pearson correlation coefficient was calculated to assess the consistency between MRAC and CTAC. For each ROI or $\mathrm{VOI}$, the relative difference of SUV in percent between
$\mathrm{PET}_{\mathrm{CT}}$ and $\mathrm{PET}_{\mathrm{MR}}$ was defined as $\left(\mathrm{SUV}_{\mathrm{MR}}-\mathrm{SUV}_{\mathrm{CT}}\right)$ / $\left(\mathrm{SUV}_{\mathrm{CT}}\right)$, and the relative difference of SUV between $\mathrm{PET}_{\mathrm{CTseg}}$ and $\mathrm{PET}_{\mathrm{MR}}$ was defined as $\left(\mathrm{SUV}_{\mathrm{MR}}-\mathrm{SUV}_{\mathrm{CTSeg}}\right)$ /

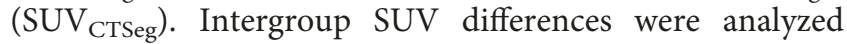
using one-way ANOVA with Bonferroni post hoc correction. The significant level was set as $P$ value less than 0.05 for all statistical analyses.

\section{Results}

5.1. MRAC Accuracy under Different MR Hardware Configurations. Images of cylinder phantom and calculated uniformities under different MR configurations are illustrated in Figure 1. Good image uniformities $(<9 \%)$ could be obtained in all images with different MR hardware configurations. Uniformities under all MR hardware configurations met the system requirement, which was defined as $<10 \%$, demonstrating accurate MRAC for MR hardware in uPMR 790 system.

5.2. Comparisons of SUVs in Main Organs. Mean SUVs of bone, liver, and lung all revealed high correlations $(>0.95)$ either between $\mathrm{PET}_{\mathrm{CT}}$ and $\mathrm{PET}_{\mathrm{MR}}$, or between $\mathrm{PET}_{\mathrm{CTSeg}}$ and $\mathrm{PET}_{\mathrm{MR}}$, demonstrating good consistencies of SUV quantifications between MRAC and CTAC (Table 2).

The mean relative differences of $\mathrm{SUV}_{\text {mean }}$ in normal bone, liver, and lung were $-18.8 \%$ (SD, 5.1\%), $-8.0 \%$ (SD, $3.8 \%$ ), and $-12.2 \%(\mathrm{SD}, 4.6 \%)$, respectively, between $\mathrm{PET}_{\mathrm{CT}}$ and $\mathrm{PET}_{\mathrm{MR}}$, and $-19.0 \%(\mathrm{SD}, 8.3 \%),-3.5 \%$ (SD, 3.6\%), and $-3.3 \%$ (SD, 6.0\%), respectively, between PET $_{\text {CTSeg }}$ and $\mathrm{PET}_{\mathrm{MR}}$. These differences were significant for all organs $(P<0.05)$. The greatest underestimation in $\mathrm{PET}_{\mathrm{MR}}$ was found in bone with a relative difference of $-18.8 \pm 5.1 \%$ between $\mathrm{PET}_{\mathrm{MR}}$ and $\mathrm{PET}_{\mathrm{CT}}$, and $-19.0 \pm 8.3 \%$ between $\mathrm{PET}_{\mathrm{MR}}$ and $\mathrm{PET}_{\mathrm{CT} \text { seg. The absolute intergroup differences }}$ between $\mathrm{PET}_{\mathrm{MR}}$ and $\mathrm{PET}_{\mathrm{CT}}$ were significantly larger than those between $\mathrm{PET}_{\mathrm{MR}}$ and $\mathrm{PET}_{\mathrm{CTSeg}}$, both in liver and in lung $(P<0.001)$, but not in bone $(P=0.893$, Table 2$)$.

5.3. Comparisons of SUVs in Lesions. Of the 28 bone lesions, both mean and maximum SUVs showed high correlations $(r>0.93)$ between PET $_{\mathrm{MR}}$ and $\mathrm{PET}_{\mathrm{CT}}$ and between $\mathrm{PET}_{\mathrm{MR}}$ and $\mathrm{PET}_{\mathrm{CTS} \text { ge }}$, demonstrating good consistencies between MRAC and CTAC (Table 2). The relative differences of $\mathrm{SUV}_{\text {mean }}$ were $-18.1 \pm 17.4 \%$ between $\mathrm{PET}_{\mathrm{MR}}$ and $\mathrm{PET}_{\mathrm{CT}}$, and $-19.3 \pm 20.4 \%$ between PET $_{\mathrm{MR}}$ and $\mathrm{PET}_{\mathrm{CTSeg}}$, while the relative differences of $\mathrm{SUV}_{\max }$ were $-13.3 \pm 10.5 \%$ between $\mathrm{PET}_{\mathrm{MR}}$ and $\mathrm{PET}_{\mathrm{CT}}$, and $-17.9 \pm 15.1 \%$ between $\mathrm{PET}_{\mathrm{MR}}$ and $\mathrm{PET}_{\mathrm{CTS} \text { eg }}$; all of these differences were statistically significant $(P<0.001$, Table 2$)$. Segmentation to CTAC had no effect on SUV quantification, as intergroup differences between PET $_{M R}$ and PET $_{C T}$ were not significantly different from

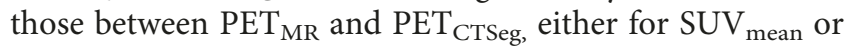
for $\operatorname{SUV}_{\text {max }}(P=0.519$ and 0.217 , respectively; Table 2$)$.

An even higher correlation was found in the group of twenty-four lesions in soft tissue. The correlation of $\mathrm{SUV}_{\text {mean }}$ and $\mathrm{SUV}_{\max }$ between $\mathrm{PET}_{\mathrm{MR}}$ and $\mathrm{PET}_{\mathrm{CT}}(r>0.98)$ and between $\mathrm{PET}_{\mathrm{MR}}$ and $\mathrm{PET}_{\mathrm{CTSeg}}(r>0.97)$ demonstrated the 


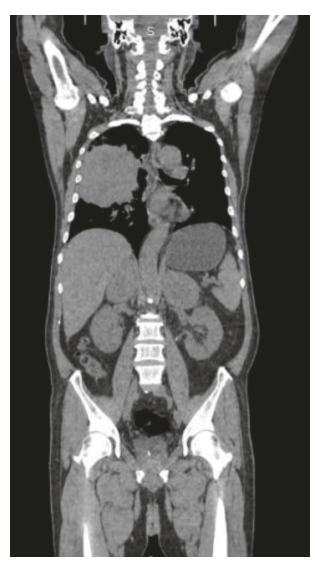

(a)

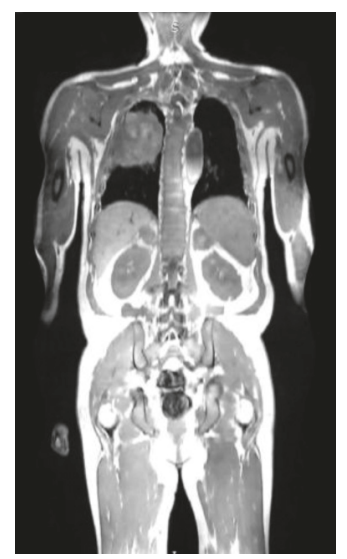

(c)

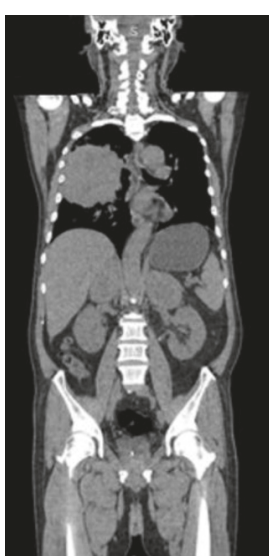

(b)

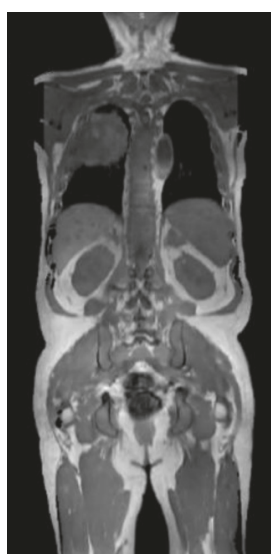

(d)

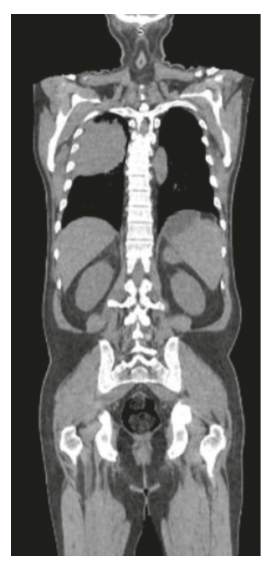

(e)

FIgure 3: Coregistration process between MR and CT images. (a) Whole-body CT image; (b) CT image without arm; (c) whole-body MR image; (d) MR image without arm; (e) CT image that coregistered to MR image space.

excellent consistencies between MRAC and CTAC (Table 2). The relative differences of $\mathrm{SUV}_{\text {mean }}$ were measured $-8.2 \pm 5.8 \%$ between PET $_{\mathrm{MR}}$ and $\mathrm{PET}_{\mathrm{CT}}$, and $-5.5 \pm 6.3 \%$ between $\mathrm{PET}_{\mathrm{MR}}$ and $\mathrm{PET}_{\mathrm{CTS} \text { eg, }}$ whereas the relative differences of $\mathrm{SUV}_{\max }$ were $-7.3 \pm 3.6 \%$ between $\mathrm{PET}_{\mathrm{MR}}$ and $\mathrm{PET}_{\mathrm{CT}}$, and $-4.4 \pm 4.0 \%$ between $\mathrm{PET}_{\mathrm{MR}}$ and $\mathrm{PET}_{\mathrm{CTSeg}}$. All these differences were statistically significant $(P<0.001)$. In addition, the absolute intergroup differences between $\mathrm{PET}_{\mathrm{MR}}$ and $\mathrm{PET}_{\mathrm{CT}}$ were significantly larger than those be-

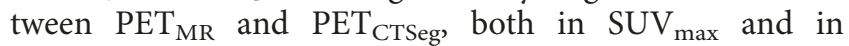

$\operatorname{SUV}_{\text {mean }}(P<0.001$, Table 2$)$, indicating a significant impact of segmentation to CT $\mu$-map on quantification differences between MRAC-based PET and CTAC-based PET.

\section{Discussion}

In this study, we validated the quantitative performance of a newly released simultaneous PET/MRI (uPMR 790, United Imaging Healthcare, Shanghai, China). The system presented good image uniformity in phantom measurements for a variety 


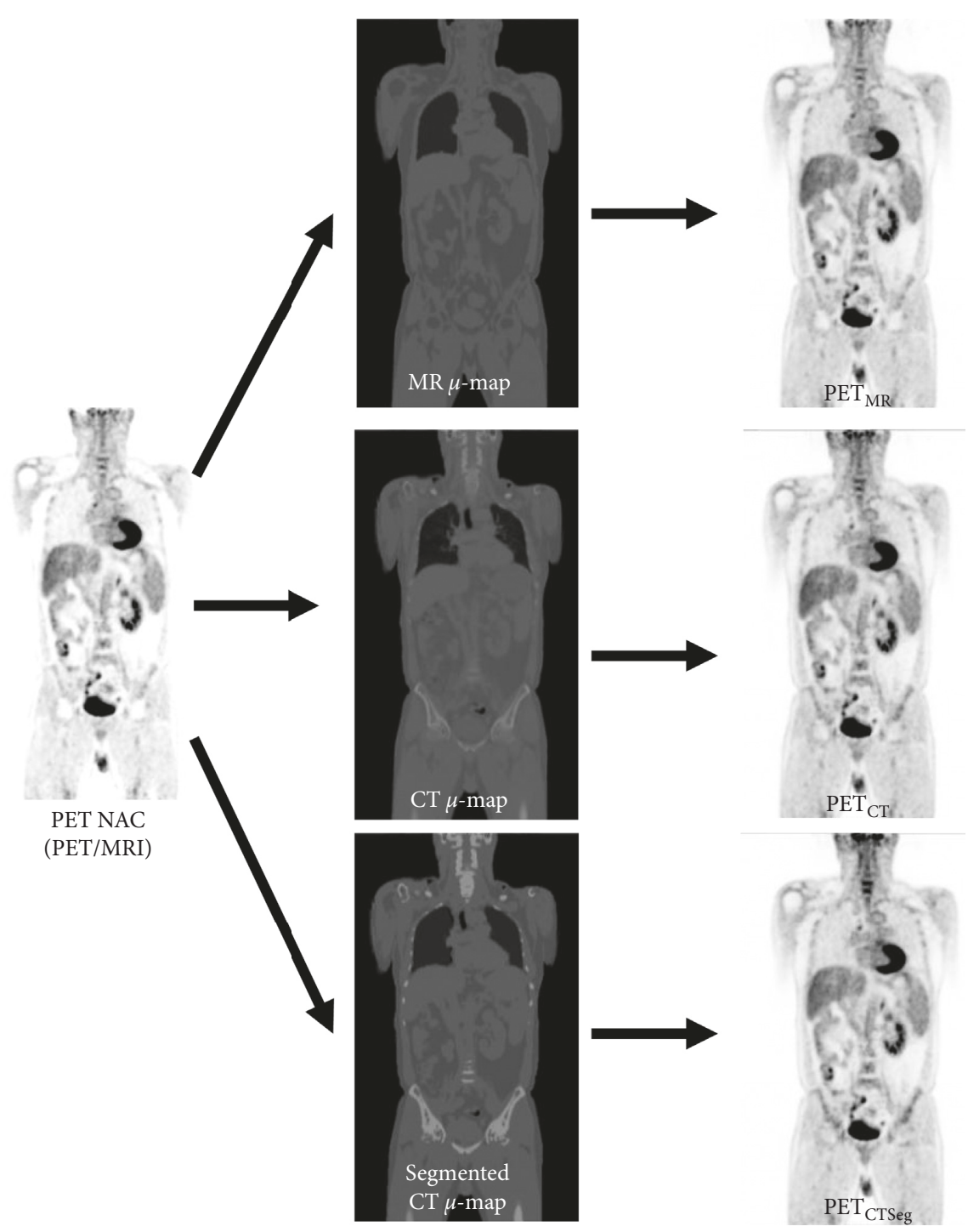

FIGURE 4: Workflow for the creation of the analyzed PET data from PET/MR corrected with MR-based (PET $\mathrm{MR}_{\text {) }}$, CT-based (PET $\mathrm{CT}$ ), and segmented CT-based AC (PET $\left.{ }_{\text {CTSeg }}\right)$.

TABLE 2: Correlations of SUVs from different PET images in major organs and in selected lesions.

\begin{tabular}{|c|c|c|c|c|c|c|c|}
\hline \multirow{2}{*}{ SUVs } & \multicolumn{3}{|c|}{$\mathrm{PET}_{\mathrm{MR}}$ and $\mathrm{PET}_{\mathrm{CT}}$} & \multicolumn{3}{|c|}{$\mathrm{PET}_{\mathrm{MR}}$ and $\mathrm{PET}_{\mathrm{CTSeg}}$} & \multirow{2}{*}{$P 3$} \\
\hline & Rho & $\mathrm{RD} 1$ & $P 1$ & Rho & $\mathrm{RD} 2$ & $P 2$ & \\
\hline \multicolumn{8}{|c|}{$\mathrm{SUV}_{\text {mean }}$ of main organs } \\
\hline Bone & 0.985 & $-18.8 \pm 5.1 \%$ & $<0.001$ & 0.967 & $-19.0 \pm 8.3 \%$ & $<0.001$ & 0.893 \\
\hline Liver & 0.973 & $-8.0 \pm 3.8 \%$ & $<0.001$ & 0.949 & $-3.5 \pm 3.6 \%$ & $<0.001$ & $<0.001$ \\
\hline Lung & 0.983 & $-12.2 \pm 4.6 \%$ & $<0.001$ & 0.959 & $-3.3 \pm 6.0 \%$ & 0.010 & $<0.001$ \\
\hline \multicolumn{8}{|c|}{ Lesions within or around bone } \\
\hline $\mathrm{SUV}_{\text {mean }}$ & 0.947 & $-18.1 \pm 17.4 \%$ & $<0.001$ & 0.938 & $-19.3 \pm 20.4 \%$ & $<0.001$ & 0.519 \\
\hline $\mathrm{SUV}_{\max }$ & 0.997 & $-13.3 \pm 10.5 \%$ & $<0.001$ & 0.975 & $-17.9 \pm 15.1 \%$ & $<0.001$ & 0.217 \\
\hline \multicolumn{8}{|c|}{ Lesions in soft tissue } \\
\hline $\mathrm{SUV}_{\text {mean }}$ & 0.981 & $-8.2 \pm 5.8 \%$ & $<0.001$ & 0.979 & $-5.5 \pm 6.3 \%$ & $<0.001$ & $<0.001$ \\
\hline $\mathrm{SUV}_{\max }$ & 0.996 & $-7.3 \pm 3.6 \%$ & $<0.001$ & 0.996 & $-4.4 \pm 4.0 \%$ & $<0.001$ & $<0.001$ \\
\hline
\end{tabular}

Note: SUV, standardized uptake value; max, maximum; $\mathrm{PET}_{\mathrm{MR}}$, MR attenuation correction-based positron emission tomography; $\mathrm{PET}_{\mathrm{CT}}$, $\mathrm{PET} \mathrm{images} \mathrm{from}$ PET/MRI that reconstructed from CT $\mu$-map; PET $_{\mathrm{CTSeg}}$, PET images from PET/MRI that reconstructed from segmented CT $\mu$-map; Rho, Pearson's correlation coefficient; RD, relative differences; $P 1$ and $P 2$ were statistical $P$ values of RD1 and RD2, respectively; $P 3$, statistical $P$ values of paired $t$-test between RD1 and RD2. 
of MR hardware configurations, which demonstrated the accuracy of MRAC for a variety of MR hardware options. A systematic clinical comparison showed that SUVs from $\mathrm{PET}_{\mathrm{MR}}, \mathrm{PET}_{\mathrm{CT}}$ and $\mathrm{PET}_{\mathrm{CTS} \text { eg }}$ were highly correlated with each other, either from a normal tissue basis or from a lesion basis. In addition, the relative differences of SUV between MRACbased PET and CTAC-based PET were generally less than $<10 \%$. These results indicated that the uPMR 790 system functioned with great promise to provide PET images with accurate quantification that was consistent with PET/CT. This system opens many opportunities for clinical and research utility for the large amount of Chinese population.

There are many technical challenges in the physical integration of simultaneous PET/MR systems. One significant challenge is the hardware components, including the patient bed, the track, and the MR coils. The strong radiofrequency (RF) wave and the rapid switching gradient system may induce noise to the PET data. On the other hand, the highly sensitive MR RF may pick up noise from the digital circuit of PET. If the coil is not accounted for in the attenuation map, significant quantification bias may occur, which can be as high as $20 \%$ [21-23]. However, in this study, good image uniformities and quantitative accuracies (bias $<10 \%$ ) were obtained in all images with different MR hardware configurations, part of which had not been discussed previously. This result was close to the data $(5.2 \%-8.2 \%)$ reported by Wollenweber et al. [22], who advocated that the bias introduced by the coil was less susceptible to cause clinically relevant errors. We did not study the bias caused by flexible surface coil, as it had been reported to be minimal [22, 24].

The segmented MRAC method classifies the body into different types of tissue; however, the classification of bone is typically underestimated and therefore not implemented into the $\mu$-map, whereas in CTAC, bone causes the highest attenuation and is easily accounted for. Therefore, significant underestimation of $\mathrm{PET}_{\mathrm{MR}}$ in bone is expected. Eiber et al. [11], Hershah et al. [12], and Heusch et al. [13] found an underestimation of SUVs $(-30.1 \%$ on average) in PET/ MR for bones. However, as these studies were performed between PET/CT and a subsequent PET/MRI, the results might be biased from the impact of the biological clearance of the tracer. When discussing the impact of different AC methods on SUV in bone or bone lesions in the same PET dataset, Martinez-Möller et al. [7] and Eiber et al. [25] found relatively lower influences, with an average underestimation of up to $-13 \%$. Seith et al. [9] observed a maximum SUV underestimation of $-17.3 \%$ in PET/MR in bone lesions. Our results regarding the underestimation of SUVs in normal bone $(-18.8 \%)$ and bone lesions $(-18.1 \%$ for $\mathrm{SUV}_{\text {mean }}$ and $-13.3 \%$ for $\mathrm{SUV}_{\text {max }}$ ) were close to these reported data. One may argue that this underestimation can lead to misinterpretation in examinations performed for assessing bone lesions. However, it should be noted that the normal uptake of FDG in normal bone is typically low, and therefore, even a small underestimation of $\mathrm{PET}_{\mathrm{MR}}$ can cause a large deviation in percentage.

For soft tissue and soft-tissue lesions, the underestimations of $\mathrm{PET}_{\mathrm{MR}}$ were relative low $(-8.0 \%$ for normal liver; $-8.2 \%$ of $\mathrm{SUV}_{\text {mean }}$ and $-7.3 \%$ of $\mathrm{SUV}_{\max }$ for soft-tissue lesions), as compared to bone tissue. Similar results were reported in previous studies which demonstrated underestimations in SUV of up to $-11 \%[9,26,27]$.

Another area with substantial underestimation of $\mathrm{PET}_{\mathrm{MR}}$ was lung $(-12.2 \pm 4.6 \%)$. Previous studies indicated that the segmentation-based MRAC might not be sufficient for reliable SUV quantification in the lung, as MRAC ignored the heterogeneity of lung intensity, probably caused by gravitation $[9,10,13,28,29]$. Both overestimation $[9,28]$ and underestimation $[10,28]$ of SUV quantification in $\mathrm{PET}_{\mathrm{MR}}$ in or adjacent to lung area had been reported. Seith et al. demonstrated an overestimation of SUV $(5.7 \pm 13.0 \%)$ in the anterior parts and an underestimation $(-14.0 \pm 15.8 \%)$ in the posterior parts of the lungs [28]. This partially supported the result of the current study, as the right lower lobe of the lung was selected for SUV measurement in this study.

One interesting finding in this study was that the relative differences of SUV compared directly between segmented MRAC-based PET $\left(\right.$ PET $\left._{M R}\right)$ and linear-CTAC-based PET $\left(\mathrm{PET}_{\mathrm{CT}}\right)$ were larger than those between $\mathrm{PET}_{\mathrm{MR}}$ and PET $_{\text {CTSeg }}$ when both attenuation corrections were segmentation based. This phenomenon was substantial in soft tissue or soft-tissue lesions $(P<0.001$; Table 2$)$. This means the segmentation nature of MRAC might have partially contributed to the deviations of SUV quantifications between $\mathrm{PET}_{\mathrm{MR}}$ and $\mathrm{PET}_{\mathrm{CT}}$. But the changes from differences of SUV between $\mathrm{PET}_{\mathrm{MR}}$ and $\mathrm{PET}_{\mathrm{CT}}$ to differences between $\mathrm{PET}_{\mathrm{MR}}$ and $\mathrm{PET}_{\mathrm{CTS} \text { eg }}$ were not significant, either in bone or in bone lesions. The underlying reason might be associated with the inability of MRAC in the identification of bone. In recent years, new techniques have been developed for MRAC with continuous LACs for bone leading to improved quantification in bone and bone lesions [30, 31]. These methods could reduce bone-related PET biases and should be used in future for whole-body MRAC.

There are several limitations in this study. First, areas of patient arms were excluded from the analysis. Since PET/CT were acquired with arms up while PET/MRI were acquired with arms down, it is very difficult to compare MRAC and CTAC in these areas. We note that none of the lesions analyzed and none of the main organs selected were in these areas. Second, ACs of brain PETs were not evaluated because the special anatomical characteristics and the different diagnostic demands in the brain were beyond the scope of this study.

\section{Conclusion}

The simultaneous whole-body PET/MRI uPMR 790 system released by the United Imaging Healthcare Corporation (Shanghai, China) functioned with accurate attenuation corrections and SUV quantifications. The SUV deviations were generally less than $10 \%$, compared to PET/CT. This system opens many opportunities for clinical and research utility for the large amount of Chinese population. However, in bone, SUV underestimations can be substantial, which may be partially due to the segmentation of the MR-based $\mu$-maps. 


\section{Data Availability}

Relevant data can be accessed through proper request, from the first author.

\section{Conflicts of Interest}

The authors declare that there are no conflicts of interest regarding this study.

\section{Acknowledgments}

This study was supported by the National Key Research and Development Program of China (grant number: 2016YFC0103908) and the National Natural Science Foundation of China (grant number: 81701730).

\section{References}

[1] C. S. Levin, S. H. Maramraju, M. M. Khalighi, T. W. Deller, G. Delso, and F. Jansen, "Design features and mutual compatibility studies of the time-of-flight PET capable GE SIGNA PET/MR system," IEEE Transactions on Medical Imaging, vol. 35, no. 8, pp. 1907-1914, 2016.

[2] G. Delso, S. Fürst, B. Jakoby et al., "Performance measurements of the Siemens mMR integrated whole-body PET/MR scanner," Journal of Nuclear Medicine, vol. 52, no. 12, pp. 1914-1922, 2011.

[3] H. Zaidi, N. Ojha, M. Morich et al., "Design and performance evaluation of a whole-body Ingenuity TF PET/MRI system," Physics in Medicine and Biology, vol. 56, no. 10, pp. 3091-3106, 2011.

[4] M. Hofmann, B. Pichler, B. Scholkopf, and T. Beyer, "Towards quantitative PET/MRI: a review of MR-based attenuation correction techniques," European Journal of Nuclear Medicine and Molecular Imaging, vol. 36, no. 1, pp. S93-S104, 2009.

[5] Y. Chen and H. An, "Attenuation correction of PET/MR imaging," Magnetic Resonance Imaging Clinics of North America, vol. 25, no. 2, pp. 245-255, 2017.

[6] I. Bezrukov, F. Mantlik, H. Schmidt, B. Schölkopf, and B. J. Pichler, "MR-based PET attenuation correction for PET/ MR imaging," Seminars in Nuclear Medicine, vol. 43, no. 1, pp. 45-59, 2013.

[7] A. Martinez-Moller, M. Souvatzoglou, G. Delso et al., "Tissue classification as a potential approach for attenuation correction in whole-body PET/MRI: evaluation with PET/CT data," Journal of Nuclear Medicine, vol. 50, no. 4, pp. 520-526, 2009.

[8] C. Brendle, H. Schmidt, A. Oergel et al., "Segmentation-based attenuation correction in positron emission tomography/ magnetic resonance," Investigative Radiology, vol. 50, no. 5, pp. 339-346, 2015.

[9] F. Seith, S. Gatidis, H. Schmidt et al., "Comparison of positron emission tomography quantification using magnetic resonance- and computed tomography-based attenuation correction in physiological tissues and lesions," Investigative Radiology, vol. 51, no. 1, pp. 66-71, 2016.

[10] D. Izquierdo-Garcia, S. J. Sawiak, K. Knesaurek, J. Narula, V. Fuster, and J. Machac, "Comparison of MR-based attenuation correction and CT-based attenuation correction of whole-body PET/MR imaging," European Journal of Nuclear Medicine and Molecular Imaging, vol. 41, no. 8, pp. 1574-1584, 2014.
[11] M. Fayad, T. Takei, M. Souvatzoglou et al., "Performance of whole-body integrated ${ }^{18} \mathrm{~F}-\mathrm{FDG}$ PET/MR in comparison to PET/CT for evaluation of malignant bone lesions," Journal of Nuclear Medicine, vol. 55, no. 2, pp. 191-197, 2014.

[12] S. Kershah, S. Partovi, B. J. Traughber, R. F. Muzic, M. D. Schluchter, and J. K. O’Donnell, “Comparison of standardized uptake values in normal structures between PET/CT and PET/MRI in an oncology patient population," Molecular Imaging and Biology, vol. 15, no. 6, pp. 776-785, 2013.

[13] P. Faulhaber, C. Buchbender, K. Beiderwellen et al., "Standardized uptake values for $\left[{ }^{18} \mathrm{~F}\right]$ FDG in normal organ tissues: comparison of whole-body PET/CT and PET/MRI," European Journal of Radiology, vol. 82, no. 5, pp. 870-876, 2013.

[14] J. Bini, D. Izquierdo-Garcia, J. Mateo, J. Machac, J. Narula, and V. Fuster, "Preclinical evaluation of MR attenuation correction versus CT attenuation correction on a sequential whole-body MR/PET scanner," Investigative Radiology, vol. 48, no. 5, pp. 313-322, 2013.

[15] M. Fayad, J. Bini, C. Calcagno, P. M. Robson, V. Mani, and Z. A. Fayad, "Attenuation correction for flexible magnetic resonance coils in combined magnetic resonance/positron emission tomography imaging," Investigative Radiology, vol. 49, no. 2, pp. 63-69, 2014.

[16] H. Arabi, O. Rager, A. Alem, A. Varoquaux, M. Becker, and H. Zaidi, "Clinical assessment of MR-guided 3-class and 4class attenuation correction in PET/MR," Molecular Imaging and Biology, vol. 17, no. 2, pp. 264-276, 2015.

[17] S. H. Keller, S. Holm, A. E. Hansen et al., "Image artifacts from MR-based attenuation correction in clinical, whole-body PET/MRI," Magnetic Resonance Materials in Physics, Biology and Medicine, vol. 26, no. 1, pp. 173-181, 2013.

[18] S. Wollenwe, S. Ambwani, A. H. R. Lonn, and D. Shanbhag, "Comparison of 4-class and continuous fat/water methods for whole-body, MR-based PET attenuation correction," IEEE Transactions on Nuclear Science, vol. 60, no. 5, pp. 3391-3398, 2013.

[19] O. Ronneberger, P. Fischer, and T. Brox, "U-net: convolutional networks for biomedical image segmentation," in Proceedings of the International Conference on Medical Image Computing and Computer-Assisted Intervention, pp. 234-241, Munich, Germany, October 2015.

[20] S. Klein, M. Staring, K. Murphy, M. A. Viergever, and J. Pluim, "Elastix: a toolbox for intensity-based medical image registration," IEEE Transactions on Medical Imaging, vol. 29, no. 1, pp. 196-205, 2010.

[21] D. H. Paulus, H. Braun, B. Aklan, and H. H. Quick, "Simultaneous PET/MR imaging: MR-based attenuation correction of local radiofrequency surface coils," Medical Physics, vol. 39, no. 1, pp. 4306-4315, 2012.

[22] S. D. Wollenweber, G. Delso, T. Deller, D. Goldhaber, M. Hüllner, and P. Veit-Haibach, "Characterization of the impact to PET quantification and image quality of an anterior array surface coil for PET/MR imaging," Magnetic Resonance Materials in Physics, Biology and Medicine, vol. 27, no. 2, pp. 149-159, 2014.

[23] L. R. MacDonald, S. Kohlmyer, C. Liu, T. K. Lewellen, and P. E. Kinahan, "Effects of MR surface coils on PET quantification," Medical Physics, vol. 38, no. 1, pp. 2948-2956, 2011.

[24] R. Kartmann, D. H. Paulus, H. Braun et al., "Integrated PET/ MR imaging: automatic attenuation correction of flexible RF coils," Medical Physics, vol. 40, no. 8, Article ID 82301, 2013.

[25] M. Eiber, A. Martinez-Möller, M. Souvatzoglou et al., "Value of a dixon-based MR/PET attenuation correction sequence for 
the localization and evaluation of PET-positive lesions," European Journal of Nuclear Medicine and Molecular Imaging, vol. 38, no. 9, pp. 1691-1701, 2011.

[26] A. Drzezga, M. Souvatzoglou, M. Eiber et al., "First clinical experience with integrated whole-body PET/MR: comparison to PET/CT in patients with oncologic diagnoses," Journal of Nuclear Medicine, vol. 53, no. 6, pp. 845-855, 2012.

[27] M. Wiesmüller, H. H. Quick, B. Navalpakkam et al., "Comparison of lesion detection and quantitation of tracer uptake between PET from a simultaneously acquiring wholebody PET/MR hybrid scanner and PET from PET/CT," European Journal of Nuclear Medicine and Molecular Imaging, vol. 40 , no. 1 , pp. 12-21, 2013.

[28] F. Seith, H. Schmidt, S. Gatidis et al., "SUV-quantification of physiological lung tissue in an integrated PET/MR-system: impact of lung density and bone tissue," PLoS One, vol. 12, no. 5, Article ID e0177856, 2017.

[29] H. R. Marshall, F. S. Prato, L. Deans, J. Theberge, R. T. Thompson, and R. Z. Stodilka, "Variable lung density consideration in attenuation correction of whole-body PET/ MRI," Journal of Nuclear Medicine, vol. 53, no. 6, pp. 977-984, 2012.

[30] M. R. Juttukonda, B. G. Mersereau, Y. Chen et al., "MR-based attenuation correction for PET/MRI neurological studies with continuous-valued attenuation coefficients for bone through a conversion from R2* to CT-Hounsfield units," Neuroimage, vol. 112, pp. 160-168, 2015.

[31] D. H. Paulus, H. H. Quick, C. Geppert et al., "Whole-body PET/MR imaging: quantitative evaluation of a novel modelbased MR attenuation correction method including bone," Journal of Nuclear Medicine, vol. 56, no. 7, pp. 1061-1066, 2015. 


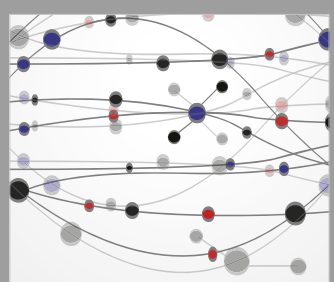

The Scientific World Journal
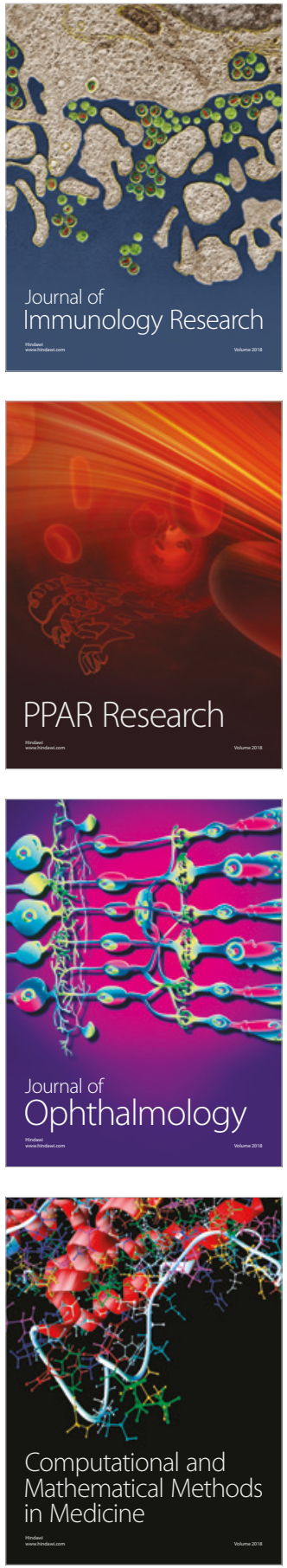

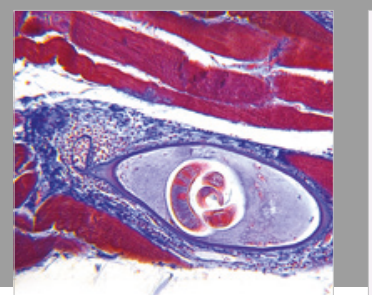

Gastroenterology Research and Practice

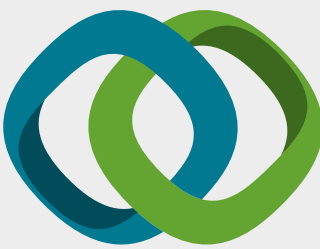

\section{Hindawi}

Submit your manuscripts at

www.hindawi.com
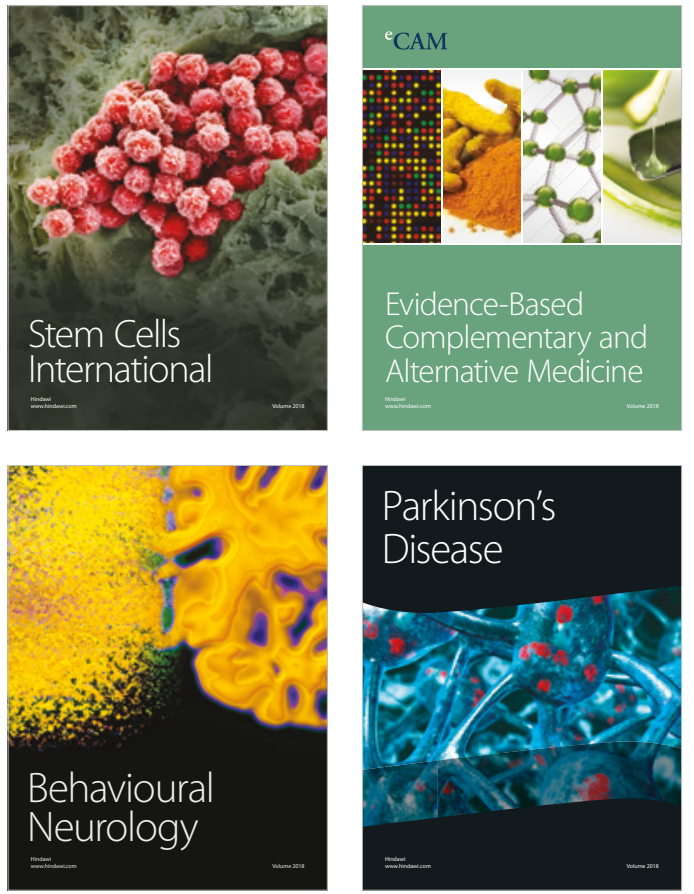

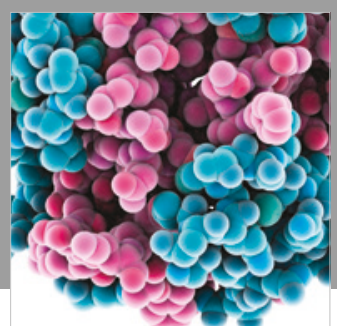

ournal of

Diabetes Research

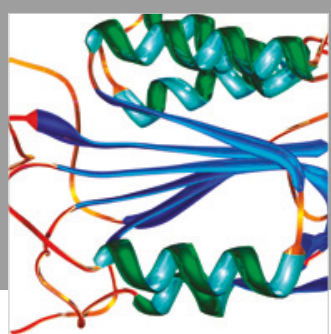

Disease Markers
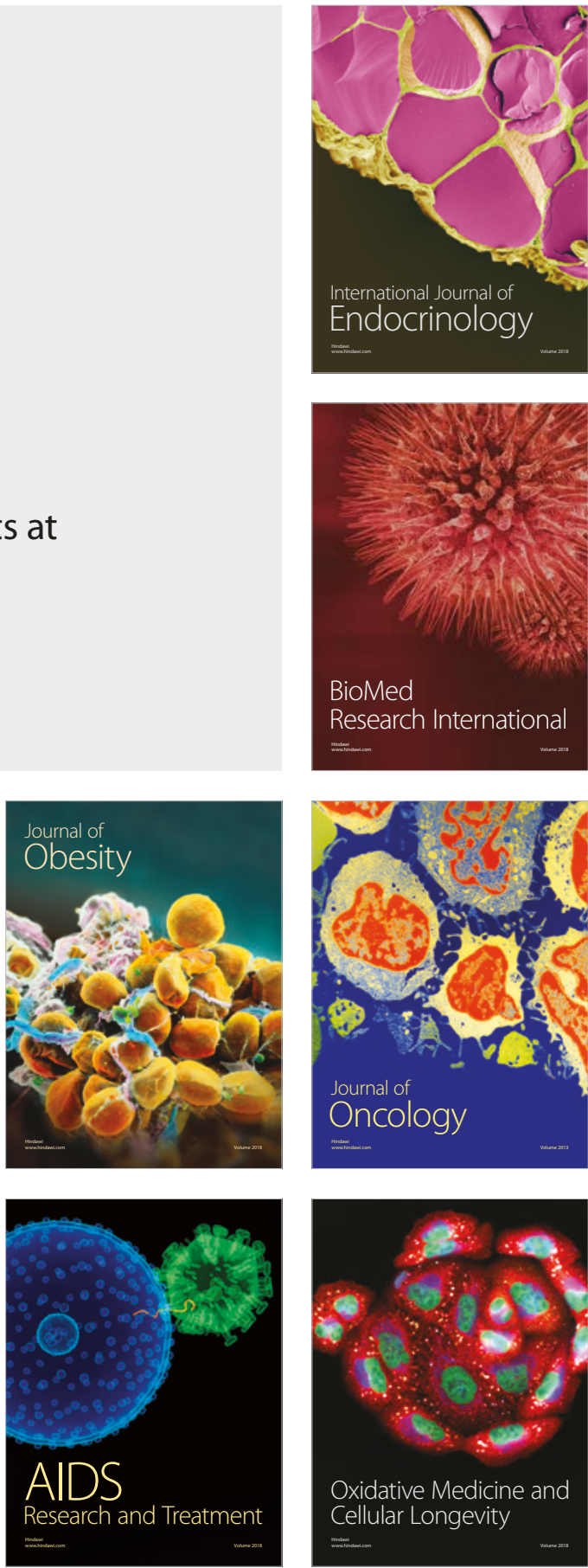